\title{
Antecedents of Approach-Avoidance Achievement Goal Adoption in Physical Education: A Longitudinal Perspective
}

\author{
Victoria E. Warburton and Christopher M. Spray \\ Loughborough University
}

\begin{abstract}
The purpose of this study was to examine the temporal pattern of pupils' approachavoidance achievement goal adoption in physical education across Key Stage 3 of secondary school. Moreover, we determined the predictive utility of implicit theories of ability and perceived competence in explaining change in achievement goals, along with the moderating influence of pupils' year group. On four occasions, over a 9-month period, 511 pupils in Years 7, 8, and 9 completed measures of perceived competence, incremental and entity beliefs, and approach-avoidance goals. Masteryapproach, mastery-avoidance, and performance-avoidance goals exhibited a linear decline over time, whereas performance-approach goals showed no significant change. Theoretical propositions regarding the antecedents of approach-avoidance goal adoption were supported. Year group was found to moderate a number of these antecedent-goal relationships. Results suggest that Year 7 is a critical time for adolescents' motivation in school physical education.
\end{abstract}

Keywords: motivation, mastery goals, performance goals, youth

The reported decline in young people's physical activity participation during the adolescent years (Pratt, Macera, \& Blanton, 1999) is particularly concerning given the well-documented benefits of participating in a regular program of physical activity (Biddle, Sallis, \& Cavill, 1998). Physical education can play an important role in the physical activity experiences of young people as it is the one physical context that encapsulates all young people. In the United Kingdom, pupils' physical education experiences in their early adolescent years are guided by the Key Stage 3 National Curriculum for Physical Education. During this Key Stage, pupils are typically in secondary schools, where they will be taught a range of activities by teachers who are specialists in physical education. How young people cognitively process their experiences of physical education during this time may affect the quality of their experiences in physical education, which in turn may influence their attitudes toward physical activity and participation beyond school

Warburton is now with the School of Education and Lifelong Learning, University of East Anglia, Norwich, United Kingdom, and Spray is with the School of Sport and Exercise Sciences, Loughborough University, Loughborough, United Kingdom. 
(Biddle, 2001; Duda, 2001; Hagger, Chatzisarantis, Culverhouse, \& Biddle, 2003).

Physical education satisfies the criteria reported in the extant literature for classification as an achievement context (Maehr, 1974). In physical education, the physical competence of a pupil is always on display and can be easily and regularly evaluated by the self and others against a standard of excellence. Pupils may strive to demonstrate competence or to avoid demonstrating incompetence (Nicholls, 1989). The transparency of competence in physical education, in conjunction with its inclusiveness of all young people aged 11-14 years, highlights the importance of understanding changes in pupils' achievement motivation and its effects on their experiences in physical education.

Achievement goal perspectives (Dweck, 1990; Elliot, 1997, 1999; Nicholls, 1989) can explain and understand pupils' motivation and behavior in an achievement situation. In the early achievement goal literature, two achievement goals were identified-mastery (approach; MAp) and performance (approach; PAp) (Dweck, 1990; Nicholls, 1989; see Duda, 2001, for a review). However, these goals focused exclusively on approach forms of motivation, that is, the need to achieve competence. More recently, Elliot $(1997,1999)$ has proposed the existence of two additional achievement goals, termed mastery avoidance (MAv) and performance avoidance (PAv) (see Elliot, 1999, 2005, for reviews), thereby creating a $2 \times 2$ achievement goal framework (Elliot \& McGregor, 2001). In this framework, MAp goals focus on developing task- or self-referent competence, whereas MAv goals focus on avoiding developing task- or self-referent incompetence. Thus, pupils may strive to do their best on a class activity in the physical education lesson, or alternatively, their striving may stem from a concern that they are unable to do the activity as well as they feel they can. Performance-approach goals focus on demonstrating normative competence, whereas PAv goals focus on avoiding demonstrating normative incompetence. Thus, pupils aim to show they are one of the best in the class at an activity, or alternatively, they are more concerned with being worse at the activity than their classmates. In both the educational and physical domains, research has shown these qualitatively different types of motivation to be associated with a differential pattern of antecedents and outcomes. These antecedents and outcomes include implicit theories of the nature of physical education ability, perceived competence, fear of failure, intrinsic motivation, anxiety, and performance (Cury, Da Fonseca, Rufo, \& Sarrazin, 2002; Cury, Elliot, Da Fonseca, \& Moller, 2006; Elliot \& Church, 1997; Elliot \& McGregor 2001; Wang, Biddle, \& Elliot, 2007). In the present research, we were concerned with two of the antecedents of achievement goal adoption, namely, perceived competence and implicit theories of ability.

\section{Perceived Competence}

Perceived competence has been a key element in research on achievement motivation (Roberts, 1984). How individuals construe their level of ability can influence the quality of their experiences in an achievement situation (Dweck, 1990; Nicholls, 1989). In Elliot's (1997, 1999) framework, perceived competence is proposed to be differentially related to the adoption of approach-avoidance achievement goals. High perceived competence is associated with the adoption of 
MAp and PAp goals, whereas low perceived competence is associated with the adoption of MAv and PAv goals. Although support for these proposed links has been found in the educational domain (Elliot \& Church, 1997), there is some discrepancy in the findings for the physical education context. Using the trichotomous conceptualization of achievement goals, Cury and colleagues (Cury et al., 2002) found support for Elliot's theoretical propositions. However, in a sample of Singaporean pupils aged 11-18 years, perceived competence demonstrated positive relations with all four approach-avoidance goals (Wang et al., 2007). Further research is needed to clarify the relations between perceived competence and approach-avoidance goals in the physical education context. Moreover, there is a need to examine this relationship over time as pupils are engaged in the physical education curriculum throughout their adolescent years. This is particularly important given that as pupils become older, their perceived competence is argued to be a more realistic appraisal of their competence (Marsh \& Craven, 1997). Longitudinal evidence in the physical education context suggests that pupil's mastery orientation and perceived competence decline with age (Papaioannou, Marsh, \& Theodorakis, 2004).

\section{Implicit Theories of Ability}

Implicit theories of ability have been documented as a key variable influencing achievement goal adoption (see Biddle, Wang, Chatzisarantis, \& Spray, 2003; Dweck, 1999; Dweck \& Leggett, 1988). Implicit theories of ability refer to an individual's view about the stability or malleability of human attributes and behaviors, such as intelligence and physical ability. Individuals who believe personal attributes and behaviors to be malleable qualities that can be improved and developed through learning are described as incremental theorists. On the other hand, those who believe personal attributes and behaviors to be fixed, stable quantities are described as entity theorists. The theory that individuals endorse influences the goals they adopt in an achievement situation, which in turn determines the motivational outcomes and patterns of behavior of the individual in that situation. Endorsing incremental beliefs leads to the adoption of mastery goals and subsequent adaptive motivational patterns regardless of perceived competence. Endorsing entity beliefs leads to the adoption of performance goals, and subsequent adaptive motivational patterns for those high in perceived competence but maladaptive motivational patterns for those low in perceived competence (see Dweck, 1999).

In a large sample of youths aged 11-19 years, Biddle and colleagues found, regardless of pupils' level of perceived competence, entity beliefs were associated with ego orientation and were a strong predictor of amotivation in physical education. On the other hand, incremental beliefs were associated with task orientation and directly predicted enjoyment. Further support in the physical education context has been provided by the work of Ommundsen (2001a, 2001b, 2003). In a series of studies examining implicit theories of ability and achievement goals, pupils who highly endorsed incremental beliefs and mastery goals reported lower levels of anxiety in physical education, higher levels of satisfaction, less use of self-handicapping strategies, and more effective self-regulation. The opposite was 
true for those pupils who endorsed entity beliefs about ability. Pupils with stronger entity beliefs and low perceived competence reported greater use of self-handicapping in physical education lessons.

Theoretical propositions in Elliot's framework (Elliot, 1999, 2005) suggest that the predictive pattern of implicit theories of ability to approach-avoidance achievement goals is associated with the definition of the goal (mastery or performance). As such, incremental beliefs are associated with MAp and MAv goals, whereas entity beliefs are associated with PAp and PAv goals. Support for this theoretical proposition has been found in both the physical and educational domains with French adolescents (Cury et al., 2002, 2006).

Implicit theories of ability are viewed as relatively stable individual differences (Dweck, 1999) and little is known about whether individuals change the implicit theory they adopt in a particular domain. In the physical education context, it seems reasonable that pupils may perceive a "ceiling" to the improvement of ability. Pupils who highly endorsed incremental beliefs in their childhood/early adolescent years may, in later adolescence, become less certain of the malleability of ability in physical education. These changes in the endorsement of implicit theories of ability will affect the experience of a pupil in physical education lessons. The shift toward endorsing entity beliefs will increase pupils' likelihood of adopting performance goals and associated maladaptive responses. Furthermore, understanding the relationship between implicit theories of ability and approachavoidance achievement goal adoption in different age groups will provide information about what age group we should target in order for interventions to have the maximum influence on pupils' motivation in physical education.

\section{The Present Research}

The purpose of the present research was to examine the temporal pattern of pupils' approach-avoidance achievement goals in physical education during Key Stage 3 of secondary school. Specifically, we examined the temporal pattern of approachavoidance achievement goal adoption in relation to implicit theories of ability and perceived competence over a 9-month period. Moreover, we examined the moderating influence of pupils' year group on the predictive utility of implicit theories of ability and perceived competence.

To our knowledge, there is little, if any, research that has longitudinally examined pupils' approach-avoidance achievement motivation in physical education using Elliot's theoretical framework. In line with theoretical propositions and previous research (Cury et al., 2002, 2006; Dweck, 1999; Elliot, 1999), it was expected that higher perceived competence would predict initial status and rate of change in MAp and PAp goals. Low perceived competence was anticipated to predict initial status and rate of change in MAv and PAv goals. Furthermore, it was expected that incremental beliefs would predict initial status and rate of change in MAp and MAv achievement goals, whereas entity beliefs would predict initial status and rate of change in PAp and PAv goals. In the absence of previous research, we did not propose hypotheses pertaining to relations between age and approachavoidance goal adoption. 


\section{Method}

\section{Participants and Procedures}

Male $(N=267)$ and female $(N=244)$ participants from physical education classes in Years 7, 8, and 9 at a state comprehensive high school in East England, United Kingdom, participated in the research. Pupils undertook units of work (6-8 lessons) in a variety of activities from the Key Stage 3 National Curriculum (e.g., football, netball, gymnastics, tennis, and cricket) and were taught in single-sex physical education classes. At the start of the research (Wave 1), participants were aged between 11 and 15 years $(M=13.18, S D=0.87$ years $)$. Although ethnicity data were not formally recorded, the vast majority of pupils were white. Procedures followed the ethical guidelines of the British Psychological Society and were approved by the ethical advisory committee at the authors' institution. Administration of questionnaires took place before a normal curriculum physical education lesson. Participants were given an explanation of how to complete each section of the questionnaire and were provided with the opportunity to ask questions. All participants were assured that the information collected would remain confidential. The questionnaire took approximately $15 \mathrm{~min}$ to complete. These procedures were repeated at 3,6, and 9 months following Wave 1 . The sections of the questionnaire were counterbalanced across measurement occasions.

\section{Measures}

Each participant completed a multisection questionnaire that provided the following information.

Personal Details. Three items relating to form group (class), date of birth, and gender comprised this section of the questionnaire. This information allowed participants to be matched on subsequent measurement occasions.

Goal Adoption. Goal adoption was assessed using the Achievement Goals Questionnaire for Sport (Conroy, Elliot, \& Hofer, 2003). Pupils responded to 12 items on a 7-point Likert scale that ranged from not at all like me (1) to very much like me (7). Three items assessed each type of goal. Sample items included, "It is important for me to perform as well as I possibly can" (MAp), "I am often concerned that I may not perform as well as I can perform" (MAv), "It is important to me to do well compared with others" (PAp), and "I just want to avoid performing worse than others" (PAv).

Implicit Theories of Ability. Participants' conceptions of the nature of ability in physical education were assessed using the Conceptions of the Nature of Athletic Ability Questionnaire Version 2 (CNAAQ-2; Biddle et al., 2003). Twelve items, assessing four subscales that reflect different representations of the nature of ability, were answered on a 5-point Likert scale that ranged from strongly disagree (1) to strongly agree (5). Sample items included, "It is difficult to change how good you are at sport/PE" (stable), "To be good at sport/PE you need to be naturally gifted" (gift), "You need to learn and to work hard to be good at sport/PE" (learning), and "If you put enough effort into it, you will always get better at sport/PE" (improvement). The CNAAQ-2 posits a hierarchical factor structure, with stable 
and gift subscales underpinning a higher order entity belief, and learning and improvement subscales underpinning a higher order incremental belief. In the current study, we were interested only in the two higher order dimensions of implicit beliefs.

Perceived Competence. Pupils' sense of competence in physical education was assessed using six items answered on a 5-point Likert scale that ranged from strongly disagree (1) to strongly agree (5). Example items included, "I am often able to successfully complete the activities I am set in PE," "I can perform tasks and skills in PE better than I used to," and "I am better at PE than others in my class."

\section{Data Analysis}

The extent and pattern of missing data across the four intervals were examined to see if any associations were evident with the substantive variables under investigation. Data were missing as a consequence of normal absences on the days of questionnaire administration. There were no significant associations between number of missed measurement occasions and initial scores on implicit beliefs, perceived competence, or goals.

All main analyses were carried out using MLwiN (version 2.0, Rasbash, Steele, Browne, \& Prosser, 2005). The software MLwiN is specifically designed to analyze multilevel data, which was reflected in the present investigation by repeated measurement occasions being nested in individuals, thus allowing both within and between levels of variance to be accounted for. In addition, MLwiN can deal with missing data and unstructured data sets, and does not require the assumption of sphericity in the data. In the current study, time and time-varying predictors represented Level 1, whereas time-invariant predictors represented Level 2 (Singer \& Willett, 2003). A series of models was examined to address the current research questions. Model A represented an unconditional means model that was used to assess the amount of between-person and within-person variance in the variables under investigation. The variance estimates produced from this model allow the intraclass correlation coefficient to be calculated. The intraclass correlation coefficient indicates how much of the total variation in the dependent variable is attributable to differences between individuals and provides the justification for using multilevel methods of data analysis. In the present research, the intraclass correlation coefficient was calculated for each of the four achievement goals, MAp (0.50), MAv (0.38), PAp (0.55), and PAv (0.47). For each goal, significant variance remained that could be explained by linear time and interindividual predictors

Model B represented an unconditional growth model that examined the initial status and rate of change for each of the variables under investigation. This model established whether there was any systematic change over time in the variables under investigation. Model $\mathrm{C}$ represented an uncontrolled effects model. These models separately examined whether implicit theories of ability or perceived competence predicted the initial status and rate of change in pupils' approachavoidance achievement goal adoption. In line with theoretical propositions, perceived competence and incremental beliefs were entered as predictors of initial status and rate of change in MAp and MAv goals. Similarly, perceived competence 
and entity beliefs were entered as predictors of initial status and rate of change in PAp and PAv goals (Dweck, 1999; Elliot, 1999).

The final model (Model D) represented a controlled effects model. These models examined the effects of implicit theories of ability or perceived competence on pupil's initial status and rates of change in approach-avoidance achievement goal adoption while controlling for the effects of pupils' year group on initial status and rate of change. In this model, Year 7 was used as the reference group to examine differences between pupils in Year 7 with those in Years 8 and 9. To simplify and clarify differences, the pupils in Years 8 and 9 were combined into one group. This was done because in similar analyses where Year 8 or 9 was used as the reference group, no significant differences between pupils in Years 8 and 9 were observed. The significant differences were observed between pupils in Years 7 and 8, and Years 7 and 9.

Model fit was assessed through examination of the deviance statistic of the model $(-2 \log L)$ and established whether adding predictors of initial status and the rate of change significantly improved model fit. Only those main effects and interaction effects for models in which a significant improvement in model fit was observed are reported (Cohen, Cohen, West, \& Aiken, 2003). The final models reported are deemed to be the most parsimonious for the data.

\section{Results}

\section{Preliminary Analyses}

At each time point, the study variables were assessed for factorial validity and structural invariance across gender and year group. The AGQ-S, CNAAQ-2, and perceived competence questionnaire demonstrated a good fit to the data (AGQ-S, 4-factor model, $\mathrm{CFI}=.970, \mathrm{SRMR}=.044 ; \mathrm{CNAAQ}-2$, hierarchical model, $\mathrm{CFI}=$ $.956, \mathrm{SRMR}=.053 ; \mathrm{PC}, 1$-factor model, $\mathrm{CFI}=.996, \mathrm{SRMR}=.018)$. Results supported previous research in the physical domain that has found the AGQ-S and CNAAQ-2 to be valid and reliable measures of approach and avoidance achievement goals and implicit theories of ability, respectively (Biddle et al., 2003; Conroy et al., 2003).

Descriptive Statistics. Descriptive statistics and internal consistency estimates were computed for each subscale at each wave of measurement and are presented in Table 1. Mean scores declined across adjacent waves of measurement for all variables except for PAp goals, entity beliefs, and perceived competence. All mean scores for all variables were above the scale midpoint at all four waves of measurement. Internal consistency estimates were assessed in relation to a criterion value of 0.70 .

\section{Main Analyses}

Temporal Patterns. The results of Model B showed that the mean level (intercept) of each achievement goal was significantly different from zero at the first wave of measurement. Mastery-approach goals (5.00) had the highest intercept and PAp goals (4.14) the lowest intercept at Wave 1. The examination of the rate 


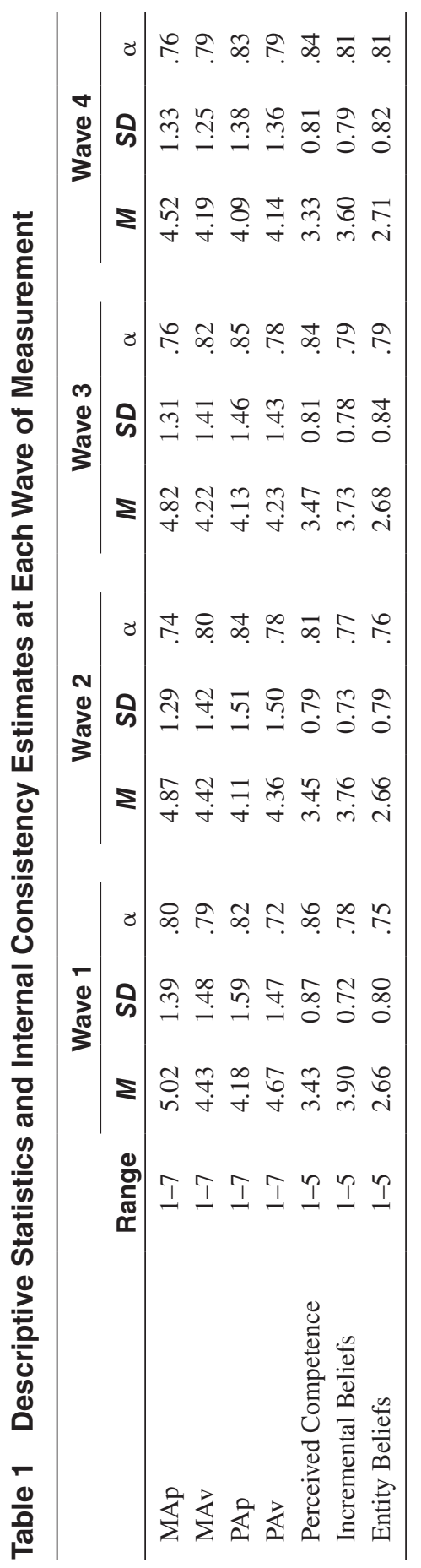


of change (slope) of the four achievement goals revealed that MAp $(\beta=-0.165$, $p<.001)$, MAv $(\beta=-0.095, p<.001)$, and PAv goals $(\beta=-0.177, p<.001)$ exhibited a significant linear decline over time. Performance-approach goals $(\beta=$ $-0.030, p>.05)$ showed no significant change over time. Linear time accounted for $10 \%$ (MAp), 11\% (MAv), and 16\% (PAv) of the within-person variance in achievement goal adoption. In addition, for MAv, PAp, and PAv goals, pupils who more highly endorsed these goals at the start of the research evidenced a slower rate of decline than pupils with lower initial levels of goal adoption. However, no association was found between initial status and rate of change in MAp goal adoption.

\section{The Effect of Ability Beliefs and Perceived Competence on Achievement Goal Adoption in Physical Education}

Predicting Change in Mastery-Approach Goal Adoption Over Time. In Model $\mathrm{C}$, the addition of either incremental beliefs or perceived competence as predictors of MAp goals revealed that both incremental beliefs $(\beta=0.767, p<.001)$ and perceived competence $(\beta=0.833, p<.001)$ positively predicted pupil's MAp goal adoption at the start of the research, but they had no effect on the rate of change over time $(p>.05)$. Table 2 presents the results of the most parsimonious model for the prediction of MAp goals and is derived from Model D, which assessed the effect of either perceived competence or incremental beliefs as predictors of MAp goal adoption when controlling for pupils' year group. The main effects from Model C remained. Controlling for year group, for a 1-point difference in perceived competence or incremental beliefs, average initial MAp goal adoption was 0.87 and 0.75 higher respectively. Year group was found to be a negative predictor of pupil's initial MAp goal adoption and a positive predictor of the rate of change in MAp goal adoption. Controlling for perceived competence or incremental beliefs, Year 7 pupils reported higher scores on MAp goals at the start of the research, and a greater decline in MAp goal adoption over time, than pupils in Years 8 and 9.

Predicting Change in Mastery-Avoidance Goal Adoption Over Time. Model C revealed that incremental beliefs $(\beta=0.482, p<.001)$ and perceived competence $(\beta=0.317, p<.001)$ separately predicted pupil's MAv goal adoption at the start of the research. Perceived competence also positively predicted the rate of change in MAv goal adoption over time $(\beta=0.083, p<.05)$. Adding perceived competence as a predictor explained $7 \%$ of the within-person variance in MAv goal adoption. Examination of Model D found no effect of year group on initial status or rate of change in MAv goal adoption.

Predicting Change in Performance-Approach Goal Adoption Over Time. In Model $\mathrm{C}$, the addition of either entity beliefs or perceived competence to the model predicting PAp goals revealed that both entity beliefs $(\beta=0.228, p<.01)$ and perceived competence $(\beta=0.718, p<.001)$ positively predicted pupil's PAp goal adoption at the start of the research. Entity beliefs also positively predicted the rate of change in PAp goal adoption over time $(\beta=0.078, p<.01)$. Adding entity beliefs as a predictor explained $5 \%$ of the within-person variance in PAp goal adoption. Table 3 presents the results of the most parsimonious model for the 


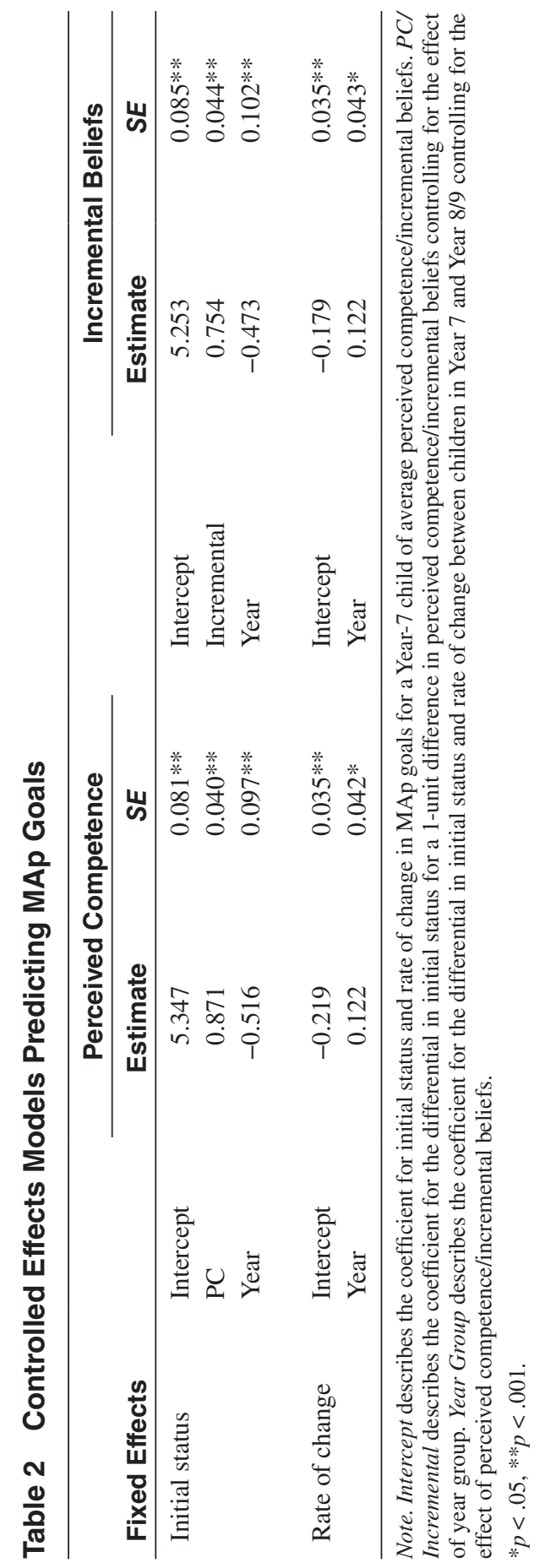


prediction of PAp goals and is derived from Model D, which assessed the effect of either perceived competence or entity beliefs as a predictor of PAp goal adoption when controlling for pupils' year group. The main effects from Model C remained. Controlling for year group, for a 1-point difference in perceived competence, average initial PAp goal adoption was 0.73 higher; for a 1-point difference in entity beliefs, average initial PAp goal adoption was 0.30 higher and average rate of change in PAp goal adoption was 0.08 higher. Year group was found to be a negative predictor of pupil's initial PAp goal adoption. Controlling for perceived competence or entity beliefs, Year-7 pupils reported higher scores on PAp goals at the start of the research, than pupils in Years 8 and 9.

Predicting Change in Performance-Avoidance Goal Adoption Over Time. In Model $\mathrm{C}$, the addition of either entity beliefs or perceived competence to the model predicting PAv goals revealed that perceived competence $(\beta=0.544, p<$ .001) positively predicted pupil's PAv goal adoption at the start of the research. Entity beliefs positively predicted the rate of change in PAv goal adoption over time $(\beta=0.147, p<.001)$. Adding entity beliefs as a predictor explained $3 \%$ of the within-person variance in PAv goal adoption. Table 4 presents the results of the most parsimonious model for the prediction of PAv goals and is derived from Model D, which assessed the effect of either perceived competence or entity beliefs as a predictor of PAv goal adoption when controlling for pupils' year group. The main effects from Model C remained. Controlling for year group, for a 1-point difference in perceived competence, average initial PAv goal adoption was 0.57 higher; for a 1-point different in entity beliefs, average rate of change was 0.15 higher. Year group was found to be a negative predictor of pupil's initial PAv goal adoption. Controlling for perceived competence or entity beliefs, Year-7 pupils reported higher scores on PAv goals at the start of the research, than pupils in Years 8 and 9.

\section{Discussion}

The decline in young people's physical activity levels is associated with their adolescent years (Biddle et al., 1998). During this time, young people's physical activity experiences in English schools are based on the Key Stage 3 National Curriculum for Physical Education. However, all too often "bad" experiences of school physical education are cited as a reason for inactivity and a failure to understand the importance of leading physically active lifestyles beyond the school curriculum (Van Wersch, Trew, \& Turner, 1992). Physical education provides the opportunity for teachers with specialist training to influence the physical activity experiences of all young people at a time when they appear most at risk for declining motivation toward physical activity. The purpose of the present research was to examine the temporal pattern of pupils' approach-avoidance achievement goals in physical education during Key Stage 3 of secondary school. Specifically, we assessed pupils' reported approach-avoidance achievement goals, implicit theories of ability, and perceived competence over a 9-month period. Moreover, we sought to delineate the moderating influence of pupils' year group on the predictive utility of implicit theories of ability and perceived competence to pupils' approach-avoidance goal adoption. 


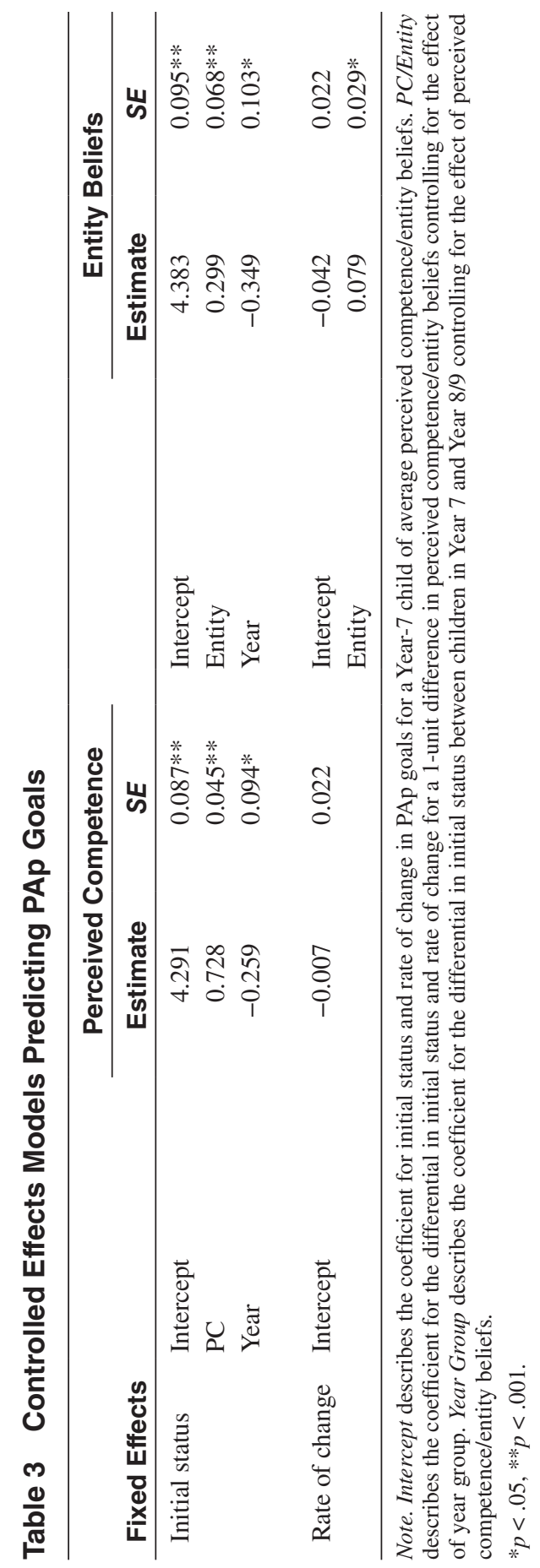




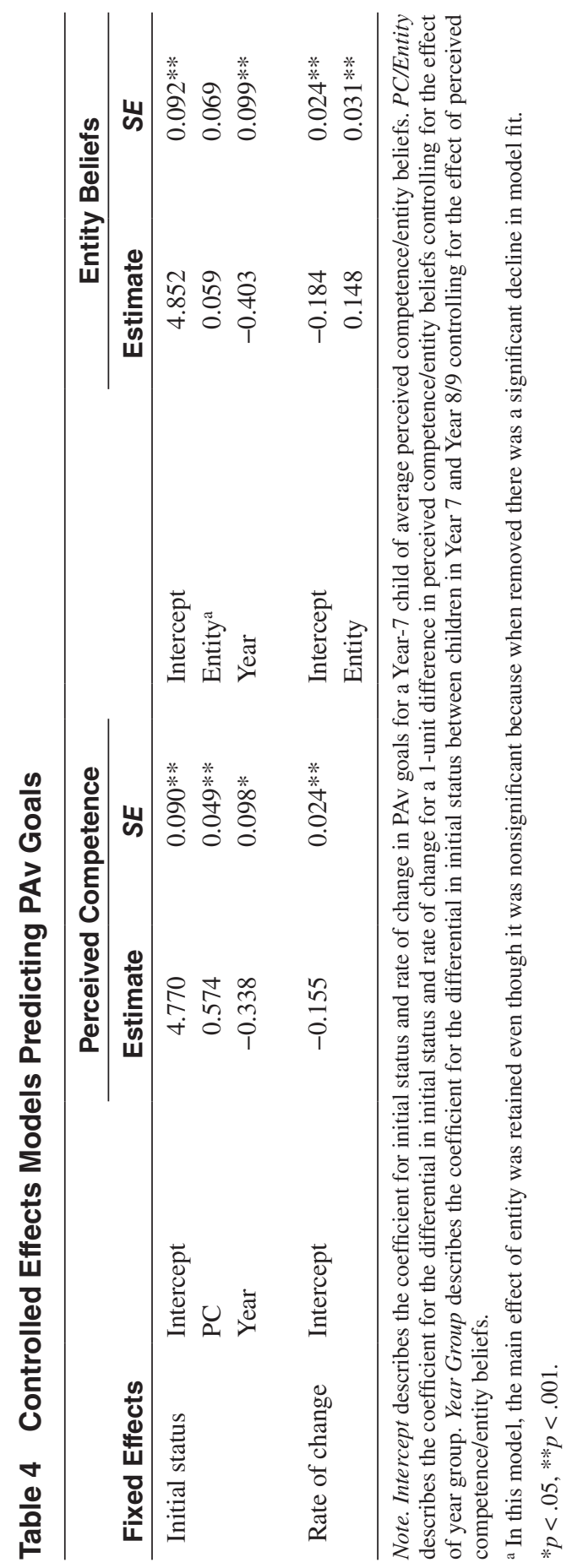




\section{Temporal Patterns}

The present research provides the first insight into the temporal pattern of pupils' approach-avoidance achievement goal adoption in physical education during Key Stage 3 of the National Curriculum for England. In general, over the period of the research, results showed a linear decline in all achievement goals, except for PAp goals. On average, in all three year groups, pupils became less concerned with self-referenced competence and mastery of tasks over time, while their focus on being competent compared with others remained stable. This may be somewhat worrisome for physical education teachers as there is an abundance of literature that identifies the adaptive consequences of adopting mastery goals rather than performance goals (Dweck, 1999; Elliot \& Church, 1997; Elliot \& McGregor, 2001). The stability of PAp goals may be a consequence of the nature of physical education lessons and the saliency it places on pupils' competence. In a physical education lesson, a pupil's physical competence is frequently on display and can be readily evaluated. For example, in a basketball lesson, the pupil who lacks the hand-eye coordination to control a basketball dribble will often be seen chasing after the ball to retrieve it. It is obvious to everyone in the lesson, both the teacher and pupils, that this pupil is unable to perform the skill, whereas others may be completing the task with ease. Pupils' experience of physical education may be detrimentally affected if the decline in all other achievement goals and particularly MAp goals continues to the point at which PAp goals become the most salient.

\section{Implicit Theories of Ability, Perceived Competence, and Approach-Avoidance Achievement Goals}

A distinct advantage of the current study over much of the previous research in the physical context was the use of a longitudinal research design. This not only enabled cross-sectional comparisons of the variables at each wave of measurement, but also an assessment of the predictive utility of key variables to changes in approach-avoidance achievement goal adoption over time. In addition, we were able to examine whether year group moderated the relationships between perceived competence, implicit theories of ability, and approach-avoidance achievement goals.

Over time, strongly endorsing entity beliefs about physical education ability can affect the achievement goal adoption of pupils' in physical education. Pupils who believed physical education ability to be a fixed, stable quantity reported an increase in the adoption of goals focusing on both normative competence and avoiding normative incompetence. These types of achievement goals, particularly the focus on incompetence, are associated with an array of maladaptive outcomes, including high levels of anxiety and worry and low levels of performance, intrinsic motivation, and persistence at tasks (Elliot \& Church, 1997; Elliot \& Conroy, 2005; Elliot \& McGregor, 2001). The present research, therefore, shows that endorsement of entity beliefs over time is associated with the adoption of potentially problematic achievement goals in physical education.

Given the central role of perceived competence in the extant literature, it is perhaps surprising that it had little effect on the temporal patterns of achievement 
goals except for that focused on avoiding self- and task-referenced incompetence (i.e., MAv goals). Pupils higher in perceived competence reported an increase in their adoption of this achievement striving over time. It is possible, however, that this finding may be a consequence of the way in which MAv goals are conceptualized in the wording of the items of the AGQ-S. The affective components in these items allow pupils who believe they are competent at physical education to be "worried," "afraid," or "concerned" about not doing as well as they previously did. Refinement and clarification of the MAv items of the AGQ-S is needed. This will enhance our understanding of these goals in the physical domain and ensure their conceptualization reflects the theorizing of Elliot (1999). In addition, the saliency of avoiding self-referenced incompetence is enhanced by the nature of physical education lessons as pupils are often completing skills and tasks they have done before to build their skill development and knowledge of an activity. Therefore, they have a reference with which to compare their current level of competence, and for some pupils, this awareness of their previous competence level may promote a "concern" or "worry" about self-referenced incompetence. Moreover, pupils may be aware of the level of the national curriculum they are working at or have achieved in previous units of work on an activity and may not want to receive a lower level than that previously achieved.

\section{The Influence of Year Group}

In addition to implicit theories of ability and perceived competence, pupils' year group accounted for some of the differences observed in the adoption of MAp, PAp, and PAv goals at the start of the research. Regardless of the level of perceived competence, incremental or entity beliefs, pupils in Year 7 reported higher levels of MAp, PAp, and PAv achievement goal adoption than pupils in Years 8 and 9. The moderating influence of year group on approach-avoidance adoption in models assessing the predictive utility of perceived competence is consistent with previous research that found perceived competence and mastery orientation to be influenced by age (Marsh, 1989; Papaioannou et al., 2004).

Of more interest, however, is the influence of year group on the change in achievement goal adoption over time. Year group was found to be a better predictor of the change in MAp goal adoption over time than was either incremental beliefs or perceived competence. Compared with pupils in Years 8 and 9 with a similar level of incremental beliefs or perceived competence, pupils in Year 7 reported a greater decline in MAp goal adoption over time. The greater focus of pupils in Year 7 on all achievement goals, including those focusing on normative competence and avoiding normative incompetence, and the greater decline in adaptive achievement goals over time, suggest that Year 7 is a critical time for pupils' motivational regulation in physical education. Results suggest that interventions designed to influence achievement goals in physical education may be most effective for pupils in Year 7. Teachers should focus on fostering beliefs that physical education ability can be improved and developed through learning, as well as facilitating pupils' perceived competence, from the start of the Key Stage 3 Curriculum rather than later in a child's school career. 
Present findings provide a rare insight into temporal relations among psychological variables that influence achievement motivation in school physical education. The information provided may help to inform interventions that aim to influence pupils' experiences of, attitudes toward, and ultimately participation in physical education and physical activity more widely. When considering the use of interventions in physical education, it is clear that there is a need to minimize the development of entity beliefs in pupils because, over time, they were associated with achievement goals focusing on normative competence and avoiding normative incompetence. As mentioned previously, these types of achievement goals are associated with several maladaptive outcomes that may negatively affect pupils' experiences in physical education. On the other hand, the present research highlighted the importance of fostering in pupils the belief that physical education ability can be improved and developed through learning and practice. These beliefs were associated with the achievement goal focused on developing self- and task-referenced competence. In the extant literature, this achievement goal is associated with adaptive outcomes, such as intrinsic motivation, persistence, selfregulated learning, and effort (Elliot \& Church, 1997; Elliot \& McGregor, 2001). However, the complexity of motivational regulation in the physical education context provides intervention research with a challenge that should not be underestimated. In the current study, incremental beliefs were also associated with the adoption of goals focusing on avoiding self- and task-referenced incompetence. Interventions that serve to impact on achievement goals through the fostering of incremental beliefs may also promote a concern with self- and task-referenced failure, which recent research suggests may be deleterious to motivation (Conroy, Kaye, \& Coatsworth, 2006).

Current findings also suggest that perceived competence may have little effect on the temporal patterns of approach-avoidance achievement goal adoption. Focusing on other antecedents of achievement striving may be more beneficial in bringing about desired changes in achievement goal adoption over time. However, the prominence of perceived competence in achievement goal approaches (Dweck, 1990; Elliot, 1997, 1999; Nicholls, 1989) suggests that this result requires further investigation. In recent years, Elliot's framework has allowed a more fine-grained analysis of achievement motivation through the inclusion of approach and avoidance components in the original mastery and performance achievement goal dichotomy. This, however, has not been accompanied by a similar analysis of perceived competence. Elliot $(1999,2005)$ distinguishes three standards by which competence can be conceived: mastery of tasks, in relation to the self, and in relation to others. In the present research, the measure of perceived competence comprised items reflecting each of these standards of competence. However, due to the relatively small number of items in the measure and the results of confirmatory factor analysis, the items were combined to form a unidimensional perceived competence factor. This unidimensional factor may not have been sufficiently sensitive to detect changes in specific approach-avoidance goals over time. A priority for future research should be the development and validation of a reliable measure of each of the standards of competence that can be employed to assess change in achievement goal adoption. This will complement the developments in 
the achievement goal literature, and we will we be able to more fully determine the contribution of perceived competence to interventions that aim to influence achievement striving in physical education.

In conclusion, the present research provides an insight into the achievement goals of pupils in physical education during a time when they are likely to experience motivational difficulties. We identified the effects of two individual difference variables to changes in pupils' approach-avoidance goals in the physical education context, but further research is needed, which addresses the limitations of the present work. The present research examined pupils once they had begun their secondary schooling. The decline observed in Year 7 may be a continuation of a downward trajectory that began before secondary school. Tracking children through primary school and into secondary school will provide an important insight into the pattern of change of motivation in physical education throughout a child's school career and identify when the downward trajectory begins. Interventions to influence pupil's motivation in physical education may actually be required before a child reaches secondary school. In this case, the employment of specialist physical education teachers with an understanding of the factors influencing children's motivation in primary schools may be warranted. In addition, the present research examined only individual difference variables in a sample from one secondary school. The amount of explained variance in achievement goal adoption was modest. Other factors, such as the perceived motivational climate in physical education, may be shown to possess greater predictive utility. A large-scale, longitudinal examination of environmental predictors of pupils' experiences of physical education will enhance our ability to explain outcomes at different levels of analysis (e.g., teacher, class, school; Marsh, Papaioannou, \& Theodorakis, 2006; Papaioannou, et al., 2004). Finally, the current research focused only on the antecedents of achievement goal adoption and therefore was unable to identify whether changes in the antecedent-goal relationship over time were manifested in changes in achievement outcomes. Physical education provides a unique opportunity to influence the physical activity experiences of all young people, and physical educators are well placed to exert a positive effect. However, achievement motivation in the physical domain is a complex process and researchers should continue their endeavors to help physical educators understand and influence pupils' motivation toward physical education and physical activity more widely.

\section{References}

Biddle, S.J.H., Sallis, J.F., \& Cavill, N. (Eds.). (1998). Young and active? Young people and health-enhancing physical activity: Evidence and implications. London: Health Education Authority.

Biddle, S.J.H. (2001). Enhancing motivation in physical education. In G.C. Roberts (Ed.), Advances in motivation in sport and exercise (pp. 101-127). Champaign, IL: Human Kinetics.

Biddle, S.J.H., Wang, C.K.J., Chatzisarantis, N.L.D., \& Spray, C.M. (2003). Motivation for physical activity in young people: Entity and incremental beliefs about athletic ability. Journal of Sports Sciences, 21, 973-989. 
Cohen, J., Cohen, P., West, S.G., \& Aiken, L.S. (2003). Applied multiple regression/correlation analysis for the behavioural sciences. Mahwah, NJ: Lawrence Erlbaum Associates.

Conroy, D.E., Elliot, A.J., \& Hofer, S.M. (2003). A 2 X 2 achievement goals questionnaire for sport: Evidence for factorial invariance, temporal stability, and external validity. Journal of Sport \& Exercise Psychology, 25, 456-476.

Conroy, D.E., Kaye, M.P., \& Coatsworth, J.D. (2006). Coaching climates and the destructive effects of mastery-avoidance achievement goals on situational motivation. Journal of Sport \& Exercise Psychology, 28, 69-92.

Cury, F., Da Fonseca, D., Rufo, M., \& Sarrazin, P. (2002). Perceptions of competence, implicit theory of ability, perception of motivational climate, and achievement goals: A test of the trichotomous conceptualization of endorsement of achievement motivation in the physical education setting. Perceptual and Motor Skills, 95, 233-244.

Cury, F., Elliot, A.J., Da Fonseca, D., \& Moller, A.C. (2006). The social-cognitive model of achievement motivation and the $2 \times 2$ achievement goal framework. Journal of Personality and Social Psychology, 90, 666-679.

Duda, J.L. (2001). Achievement goal research in sport: Pushing the boundaries and clarifying some misunderstandings. In G.C. Roberts (Ed.), Advances in motivation in sport and exercise (pp. 129-182). Champaign, IL: Human Kinetics.

Dweck, C.S. (1990). Self-theories and goals: Their role in motivation, personality, and development. In R. Dienstbier (Ed.), Nebraska symposium on motivation 1990. Perspectives on motivation (Vol. 38, pp. 199-235). Lincoln, NE: University of Nebraska Press.

Dweck, C.S. (1999). Self theories: Their role in motivation, personality, and development. Philadelphia, PA: Psychology Press.

Dweck, C.S., \& Leggett, E.L. (1988). A social-cognitive approach to motivation and personality. Psychological Review, 95, 256-273.

Elliot, A.J. (1997). Integrating the "classic" and "contemporary" approaches to achievement motivation: A hierarchical model of approach and avoidance achievement motivation. In M.L. Maehr \& P.R. Pintrich (Eds.), Advances in motivation and achievement (Vol. 10, pp. 143-179). Greenwich, CT: JAI Press.

Elliot, A.J., \& Church, M.A. (1997). A hierarchical model of approach and avoidance achievement motivation. Journal of Personality and Social Psychology, 72, 218-232.

Elliot, A.J. (1999). Approach and avoidance motivation and achievement goals. Educational Psychologist, 34, 169-189.

Elliot, A.J., \& McGregor, H.A. (2001). A 2 X 2 achievement goal framework. Journal of Personality and Social Psychology, 80, 501-519.

Elliot, A.J. (2005). A conceptual history of the achievement goal construct. In A.J. Elliot \& C.S. Dweck (Eds.), Handbook of competence and motivation (pp. 52-72). New York: Guildford Press.

Elliot, A.J., \& Conroy, D.E. (2005). Beyond the dichotomous model of achievement goals in sport and exercise psychology. Sport \& Exercise Psychology Review, 1, 17-25.

Hagger, M.S., Chatzisarantis, N.L.D., Culverhouse, T., \& Biddle, S.J.H. (2003). The processes by which perceived autonomy support in physical education promotes leisuretime physical activity intentions and behavior: A trans-contextual model. Journal of Educational Psychology, 95, 784-795.

Maehr, M.L. (1974). Culture and achievement motivation. The American Psychologist, 29, 887-896.

Marsh, H.W. (1989). Age and Sex Effects in Multiple Dimensions of Self-Concept - Preadolescence to Early Adulthood. Journal of Educational Psychology, 81, 417-430.

Marsh, H.W., \& Craven, R. (1997). Academic self-concept: Beyond the dustbowl. In G. Phye (Ed.), Handbook of classroom assessment: Learning, achievement and adjustment. San Diego, CA: Academic Press. 
Marsh, H.W., Papaioannou, A., \& Theodorakis, Y. (2006). Causal ordering of physical selfconcept and exercise behavior: Reciprocal effects model and the influence of physical education teachers. Health Psychology, 25, 316-328.

Nicholls, J.G. (1989). The competitive ethos and democratic education. Cambridge, MA: Harvard University Press.

Ommundsen, Y. (2001a). Pupils' affective responses in physical education classes: the association of implicit theories of the nature of ability and achievement goals. European Physical Education Review, 7, 219-242.

Ommundsen, Y. (2001b). Self-handicapping strategies in physical education classes: the influence of implicit theories of the nature of ability and achievement goal orientations. Psychology of Sport and Exercise, 2, 139-156.

Ommundsen, Y. (2003). Implicit theories of ability and self-regulation strategies in physical education classes. Educational Psychology, 23, 141-157.

Papaioannou, A., Marsh, H.W., \& Theodorakis, Y. (2004). A multilevel approach to motivational climate in physical education and sport settings: An individual or a group level construct? Journal of Sport \& Exercise Psychology, 26, 90-118.

Pratt, M., Macera, C.A., \& Blanton, C. (1999). Levels of physical activity and inactivity in children and adults in the United States: current evidence and research issues. Medicine and Science in Sports and Exercise, 31, S526-S533.

Rasbash, J., Steele, F., Browne, W., \& Prosser, B. (2005). A user's guide to MLwiN version 2.0. United Kingdom: University of Bristol.

Roberts, G.C. (1984). Achievement motivation in children's sport. In J.G. Nicholls \& M.L. Maehr (Eds.), Advances in motivation and achievement: The development of achievement motivation (Vol. 3, pp. 251-281). Greenwich, CT: JAI Press.

Singer, J.D., \& Willett, J.B. (2003). Applied longitudinal data analysis: Modeling change and event occurrence. New York: Oxford University Press.

Van Wersch, A., Trew, K., \& Turner, I. (1992). Post-primary school pupils' interest in physical education: Age and gender differences. The British Journal of Educational Psychology, 62, 56-72.

Wang, C.K.J., Biddle, S.J.H., \& Elliot, A.J. (2007). The 2x2 achievement goal framework in a physical education context. Psychology of Sport and Exercise, 8, 147-168. 